\title{
GMR
}

\section{Are Epichloë endophytes specific to Elymus grass hosts?}

\author{
H. Song, Q.Y. Song, X.Z. Li and Z.B. Nan \\ Key Laboratory of Grassland Agro-Ecosystems, \\ College of Pastoral Agriculture Science and Technology, \\ Lanzhou University, Lanzhou, China \\ Corresponding author: Z.B. Nan \\ E-mail: zhibiao@Izu.edu.cn
}

Genet. Mol. Res. 14 (4): 17463-17471 (2015)

Received August 6, 2015

Accepted October 2, 2015

Published December 21, 2015

DOI http://dx.doi.org/10.4238/2015.December.21.17

\begin{abstract}
Epichloë endophytes are widely distributed mutualists of cool-season grasses and can protect their hosts against biotic and abiotic stresses. Previous studies have shown that Epichloë endophytes are specific to their grass hosts in tall Festuca and Lolium species. However, no systematic analysis exists of host specificity of asexual Epichloë endophytes and Chinese Elymus species. We analyzed the phylogenetic relationships between Chinese Elymus species and their diploid donor Hordeum species, using their corresponding Epichloë endophyte sequences. We found that 1) the maternal donor of the Chinese Elymus species was the Chinese Pseudoroegneria (St genome) or Hordeum ( $\mathrm{H}$ genome); and 2) Chinese Hordeum species probably contained two species of Epichloë endophytes. One Epichloë endophyte was also present in a North American Elymus species. The other Epichloë endophyte was found in a Chinese Elymus species. Our results indicate that Epichloë endophytes isolated from Elymus species did not show grass-host specificity. 3) Plant hybridization could probably transform endophyte-free plants (E-) to endophyte-infected plants $(E+)$. Based on
\end{abstract}


our data, we formulate hypotheses about which Epichloë endophytes were spread via plant hybridization.

Key words: Elymus species; Epichloë endophyte; Hordeum species; Host specificity; Maternal donor; Phylogeny

\section{INTRODUCTION}

Forage grasses (Poaceae) often harbor endophytic fungi of the genus Epichloë (Clavicipitaceae, Ascomycota) (Clay and Schardl, 2002). Asexual Epichloë endophytes inhabit the intercellular spaces of their grass hosts and depend entirely on the host plant for propagation through its seeds or vegetative structures (Schardl, 2010). It is well documented that asexual Epichloë endophytes can spread vertically by infecting the seeds of the host plant without overt symptoms of disease (Clay and Schardl, 2002; Selosse and Schardl, 2007). Sexual Epichloë endophytes are horizontally transmitted and can develop a stroma from which contagious spores are released. Sexual Epichloë endophytes may induce symptoms of choke disease, in which the infected host grass aborts the development of reproductive structures (Johnson et al., 2013). However, not all sexual Epichloë endophytes induce choke symptoms; some sexual Epichloë endophytes are designated non-choke-inducing types (Clay and Schardl, 2002) and, under certain conditions, these species can have a positive effect on the grass host (Clay and Schardl, 2002; Schardl, 2010).

Many members of the grass family Poaceae have coevolved with Epichloë endophytes, including asexual morphs (Schardl et al., 1997). In most cases, Epichloë endophytes are only found in association with grasses of the subfamily Pooideae, including the tribes Aveneae, Brachypodieae, Bromeae, Meliceae, Poeae, Stipeae, and Triticeae, with the majority systematically colonizing their hosts and being transmitted vertically to the next generation (Schardl and Philips, 1997). Epichloë endophytes exhibit coevolutionary patterns at the tribe or genus level, but the significance of this evolutionary trend at the species or subspecies level of the grass host has not been fully investigated. A broad-range study on asexual Epichloë endophytes in grass hosts found that they did not coevolve or codiverge (Schardl et al., 2008). However, the relationships between Chinese Elymus species and related asexual Epichloë endophytes have not been analyzed.

Elymus L. is the largest genus of grasses in the tribe Triticeae (Poaceae), with approximately 150 perennial species distributed across temperate zones throughout most of the worldwide (Dewey, 1984), except for Africa and Antarctica (Helfgott and Mason-Gamer, 2004). An analysis of the chromosomes of tetraploid, hexaploid, and octoploid Elymus species indicated that they have five potential diploid donors: Pseudoroegneria (St genome), Hordeum (H genome), Agropyron (P genome), Australopyrum (W genome), and an unknown species (Y genome) (Dewey, 1984; Jensen, 1990; Torabinejad and Mueller, 1993). A recent report indicated that Pseudoroegneria has no endophytes; however, many asexual Epichloë endophytes and Epichloë bromicola are found in Hordeum and Elymus species from China, respectively (Card et al., 2014; Song and Nan, 2015). For the present study, we collected polyploid Elymus and diploid Hordeum species in western China and isolated their Epichloë endophytes. Additionally, sequences of North American polyploid Elymus and diploid Hordeum species and their related Epichloë endophytes were obtained from GenBank. The goal of our study was to determine whether the same Epichloë endophytes were present in Chinese and American Elymus and Hordeum species. 


\section{MATERIAL AND METHODS}

\section{Ethics statement}

No specific permissions were required for this study as we collected a limited number of seeds and stalks from native grassland that was not privately owned or protected. Our field study did not involve any endangered or protected species.

\section{Plant materials}

In total, 398 individuals from four diploid Hordeum species were sampled from four populations in western China. In addition, sequences of North American polyploid Elymus and diploid Hordeum species and their related Epichloë endophytes were obtained from GenBank. (Table 1).

\begin{tabular}{|c|c|c|c|c|c|c|c|}
\hline Isolate & Host species & Host tribe & $\begin{array}{l}\text { Accession No. } \\
\text { of endophyte }\end{array}$ & Collection locality & $t r n L-F$ & tubB & tefA \\
\hline Epichloë amarillas & Elymus virginicus & Triticeae & E1087 & North America (USA) & AF519144 & AF457478 & AF457518 \\
\hline Epichloë clarkii & Elymus hystrix & Triticeae & ATCC201556 & North America (USA) & AF519139 & AF250744 & AF457500 \\
\hline Epichloë elymi & Elymus virginicus & Triticeae & ATCC201553 & North America (USA) & AF519145 & AF062428 & AF457498 \\
\hline Epichloë elymi & Elymus hystrix & Triticeae & ATCC201557 & North America (USA) & EF396985 & AF250745 & AF457501 \\
\hline Epichloë elymi & Elymus canadensis & Triticeae & ATCC201551 & North America (USA) & EU119363 & L06962 & AF231209 \\
\hline Epichloë elymi & Elymus virginicus & Triticeae & MP-III & North America (USA) & AF519143 & L78273 & AF231208 \\
\hline Epichloë bromicola & Elymus excelsus & Triticeae & NI_201201 & Asia (China) & KF905187 & KJ585731 & KJ585716 \\
\hline Epichloë bromicola & Elymus excelsus & Triticeae & NI_201203 & Asia (China) & KF905189 & KJ585732 & KJ585717 \\
\hline Epichloë bromicola & Elymus tibeticus & Triticeae & NI_201206 & Asia (China) & KF905192 & KJ585733 & KJ585718 \\
\hline Epichloë bromicola & Elymus dahuricus & Triticeae & NI_201208 & Asia (China) & KF905194 & KJ585734 & KJ585720 \\
\hline Epichloë bromicola & Elymus tangutorum & Triticeae & NI_201210 & Asia (China) & KF905196 & KJ585736 & KJ585721 \\
\hline Epichloë bromicola & Elymus tangutorum & Triticeae & NI_201213 & Asia (China) & KF905199 & KJ585737 & KJ585722 \\
\hline Epichloë bromicola & Elymus excelsus & Triticeae & NI_201214 & Asia (China) & KF905200 & KJ585738 & KJ585723 \\
\hline Epichloë bromicola & Elymus tangutorum & Triticeae & NI_201216 & Asia (China) & KF905202 & KJ585739 & KJ585724 \\
\hline Epichloë bromicola & Elymus excelsus & Triticeae & NI_201218 & Asia (China) & KF905204 & KJ585740 & KJ585725 \\
\hline Epichloë bromicola & Elymus tangutorum & Triticeae & NI_201221 & Asia (China) & KF905207 & KJ585741 & KJ585726 \\
\hline Epichloë bromicola & Elymus dahuricus var. cylindricus & Triticeae & NI_201222 & Asia (China) & KF905208 & KJ585742 & KJ585727 \\
\hline Epichloë bromicola & Elymus nutans & Triticeae & NI_201302 & Asia (China) & KF905216 & KJ585743 & KJ585728 \\
\hline Epichloë bromicola & Elymus dahuricus & Triticeae & NI_201306 & Asia (China) & KF905220 & KJ585744 & KJ585729 \\
\hline Epichloë bromicola & Elymus dahuricus var. cylindricus & Triticeae & NI_201308 & Asia (China) & KF905222 & KJ585745 & KJ585730 \\
\hline Epichloë sp & Hordeum bogdanii & Triticeae & $\mathrm{N} 100$ & Asia (China) & KP087958 & KP087966 & KP087962 \\
\hline Epichloë sp & Hordeum jubatum & Triticeae & N133 & Asia (China) & KP087961 & KP087968 & KP087965 \\
\hline Epichloë sp & Hordeum jubatum & Triticeae & N13 & Asia (China) & KP087960 & KP087967 & KP087964 \\
\hline Epichloë sp & Hordeum roshevitzii & Triticeae & N11 & Asia (China) & KP087959 & KP087969 & KP087963 \\
\hline
\end{tabular}

Bolded entries represent sequences generated in this study.

\section{DNA extraction, polymerase chain reaction (PCR) amplification, and sequencing}

Genomic DNAs of diploid Hordeum species were extracted from fresh leaves using a plant DNA isolation kit (TaKaRa, Dalian, China). Endophyte genomic DNAs from mature plants (Schardl et al., 2013) were extracted using the HP fungal DNA kit (Omega, Beijing, China).

The chloroplast non-coding region trnL-F was amplified from diploid Hordeum species using primers described previously (Taberlet et al., 1991; Zhu et al., 2013). Intron-rich portions of the housekeeping genes $\beta$-tubulin $(t u b B)$ and translation elongation factor 1- $\alpha($ tef $A$ ) were amplified from the endophytes using the PCR protocols described by Moon et al. (2002). 
PCR products were cloned into the pMD18-T vector (TaKaRa, Dalian, China) following the manufacturer instructions. Three to five positive clones per accession were sequenced by TaKaRa Biotechnology Co. Ltd (Dalian, China). Sequences were deposited in GenBank (Table 1) under accession Nos. KP087958-KP087961 (trnL-F), KP087966-KP087969 (tubB), and KP087962KP087965 (tefA).

\section{Phylogenetic analyses}

Sequences were aligned using the MAFFT 7.0 software (Katoh and Standley, 2013) and adjusted using the Genedoc software (Nicholas and Nicholas, 1997). Phylogenetic relationships were estimated using maximum parsimony (MP) and maximum likelihood (ML) methods in the PAUP 4.0b10 package (Swofford, 2002) with 1000 replications for each. Endophyte phylogenetic trees were constructed using $t u b B$ and tef $A$ intron sequences after they were concatenated using the SequenceMatrix software (Vaidya et al., 2011). MP trees were estimated using a heuristic search based on tree bisection-reconnection (TBR) branch swapping and 100 random-addition replicates. The ML estimate used the best-fitting model of sequence evolution in MrModeltest (Nylander, 2004). This was estimated to be $\mathrm{K} 80+\mathrm{G}$ for the $t u b B+t e f A$ endophyte dataset and HKY for the grass trnL-F dataset. Alignment gaps were treated as missing information. Nucleotide substitutions were unordered and unweighted.

\section{RESULTS AND DISCUSSION}

\section{Grass chloroplast trnL-F phylogeny}

The parsimony analysis of $t r n \mathrm{~L}-\mathrm{F}$ sequences yielded a tree with a length of 95 (consistency index $[\mathrm{Cl}]=0.968$, rescaled consistency index $[\mathrm{RI}]=0.967$ ). Phylogenetic relationships among North American and Chinese Elymus species are shown in Figure 1. Only the data from the MP tree are shown here. The trnL-F phylogenetic tree of Chinese and North American Elymus and Chinese Hordeum species identified three clades (Figure 1). Clade I contained two sequences from the North American Elymus hystrix and Chinese E. tangutorum. Clade II was weakly supported (60/69) and contained two collections from the North American E. canadensis and Chinese E. dahuricus var. cylindricus. The species constituting clades I and II support the hypothesis that Elymus specieshas had multiple dispersal events. The rich diversity of Elymus species in central Asia suggests that it could be the center of origin for Elymus species (Lu and Salömon, 1992). Therefore, it is not surprising that North American and Chinese Elymus species may have a common ancestor.

As described above, five basic genomes are found in Elymus and are derived from Pseudoroegneria (St genome), Hordeum (H genome), Agropyron ( $\mathrm{P}$ genome), Australopyrum (W genome), and an unknown species (Y genome) (Torabinejad and Mueller, 1993; Sun, 2014). The St genome is a fundamental genome that originated in Elymus and is found in all Elymus species (Sun, 2014). North American Elymus species contain the St and H genomes (MasonGamer, 2013), whereas Asian Elymus species contain the $Y$ genome (Lu, 1993). Here we found that Elymus species from clades I and II contained the St and $\mathrm{H}$ genomes: E. hystrix, StH; E. tangutorum, StStH; E. canadensis, StH; E. dahuricus var. cylindricus, StHY (Mason-Gamer et al., 2010; Mason-Gamer, 2013). The chloroplast genome typically represents the maternal parent in angiosperms, and previous studies have found that North American Pseudoroegneria species (St 
genome) are the maternal donor to North American Elymus species (Mason-Gamer and Orme, 2002; Sun, 2002, 2014). We found that the maternal parent of Chinese E. tangutorum (KF905207) and E. dahuricus var. cylindricus (KF905208) is also a North American Pseudoroegneria species (St genome). Interestingly, other Chinese collections of E. tangutorum (KF905199, KF905196 and KF905202) and E. dahuricus var. cylindricus (KF905222) did not cluster with North American Elymus species, suggesting that they probably had different maternal donors.

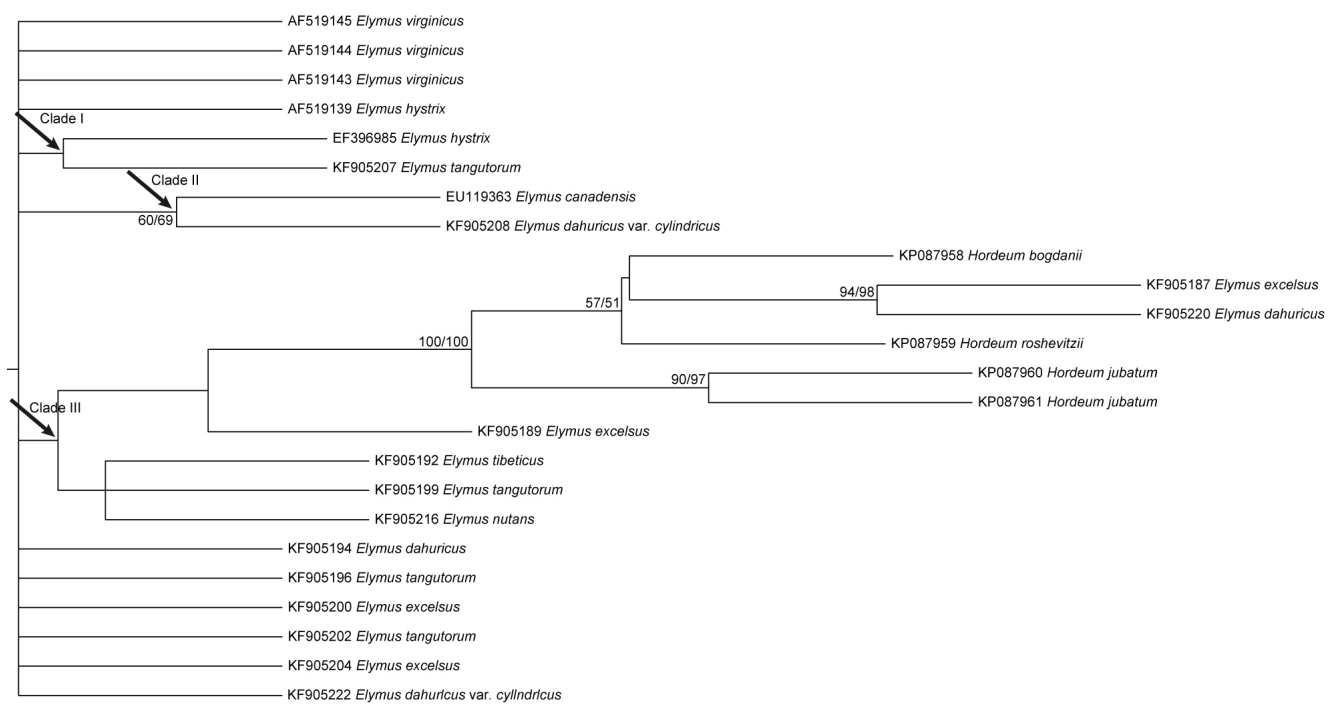

Figure 1. Chloroplast trnL-F maximum-parsimony tree. Bootstrap support values were calculated from 1000 replications. Branches with bootstrap values $>50 \%$ are shown. MP bootstrap values are listed first, followed by ML bootstrap values. Arrows indicate the clades described in the text.

Clade III contained 10 samples from Chinese Elymus and Hordeum species, indicating that a Chinese Pseudoroegneria species (St genome) was the maternal donor to some of the Chinese Elymus species. In addition, six Chinese Elymus species were unresolved in the trnL-F phylogeny. The maternal donor of these Elymus species may have been a Chinese Pseudoroegneria (St genome) or Hordeum (H genome). However, this result needs further study. Nevertheless, it is certain that Chinese Elymus species did not have a single maternal donor, as do North American Elymus species. Instead, Chinese Elymus species might have had a number of maternal donors, including Pseudoroegneria (St genome) or Hordeum (H genome).

\section{Endophytic tubB + tefA tree}

The MP analysis of $t u b B+t e f \mathrm{~A}$ yielded a tree with a length of 136 (Figure 2; $\mathrm{Cl}=0.9485$; $\mathrm{RI}=0.9663)$. Topology of the ML tree was slightly different from that of the MP tree, but both methods recovered one clade (Figure 2, shaded branches).

Both MP and ML phylogenetic trees identified a clade including sexual Epichloë endophytes from North American E. canadensis, E. hystrix, and E. virginicus, and asexual Epichloë endophytes from Chinese Hordeum jubatum and $H$. roshevitzii (Figure 2), suggesting that the endophytes in these Elymus and Hordeum species have the same origin. Previous studies have 
shown that grass-endophyte interactions are highly specific (Ekanayake et al., 2012; Gundel et al., 2012; Karimi et al., 2012), and that each designated endophyte grouping generally has a high degree of host specificity (Schardl et al., 1997). As anticipated, Epichloë coenophiala was found in Continental hexaploid tall fescue host plants, including the tetraploid maternal progenitor of Continental hexaploid tall fescue (Ekanayake et al., 2012). Karimi et al. (2012) found that the host specificity of asexual Epichloë endophytes from Festuca arundinacea, F. pratensis, and Lolium perenne was consistent in geographically distant host grasses of the same species. However, Card et al. (2014) analyzed endophytes isolated from Hordeum and Elymus species and found that most endophytes cluster according to geographical regions rather than host species. Our results were not consistent with either of the above findings. The host specificity of endophytes from Chinese Hordeum species was not necessarily consistent between multiple samples from a particular host species, and they were not consistent according to geographical location of the collection (Figure 2). Although there are differences between the MP and ML tree topologies, we noted that an asexual Epichloë endophyte isolated from Chinese $H$. bogdanii had the same origin as asexual isolates from some of the Chinese Elymus species, indicating that Chinese Hordeum species carry a variety of Epichloë endophytes. Asexual Epichloë endophytes from Chinese Hordeum species have a common ancestor with sexual Epichloë sexual endophytes from North American Elymus species. Additionally, asexual Epichloë endophytes from Chinese Hordeum and Elymus species have the same origin. Isoenzyme analyses suggest that Eurasian and American StH-genome tetraploid Elymus species may have originated independently from different $\mathrm{H}$-genome donors (Jaaska, 1992). Genomic (Wang and Hsiao, 1986), and karyotypic analyses (Linde-Laursen et al., 1994) suggest that Old and New World taxa may be of different origin, and therefore, Chinese and North American Elymus species may carry different endophytes because they have different Hordeum species donors.

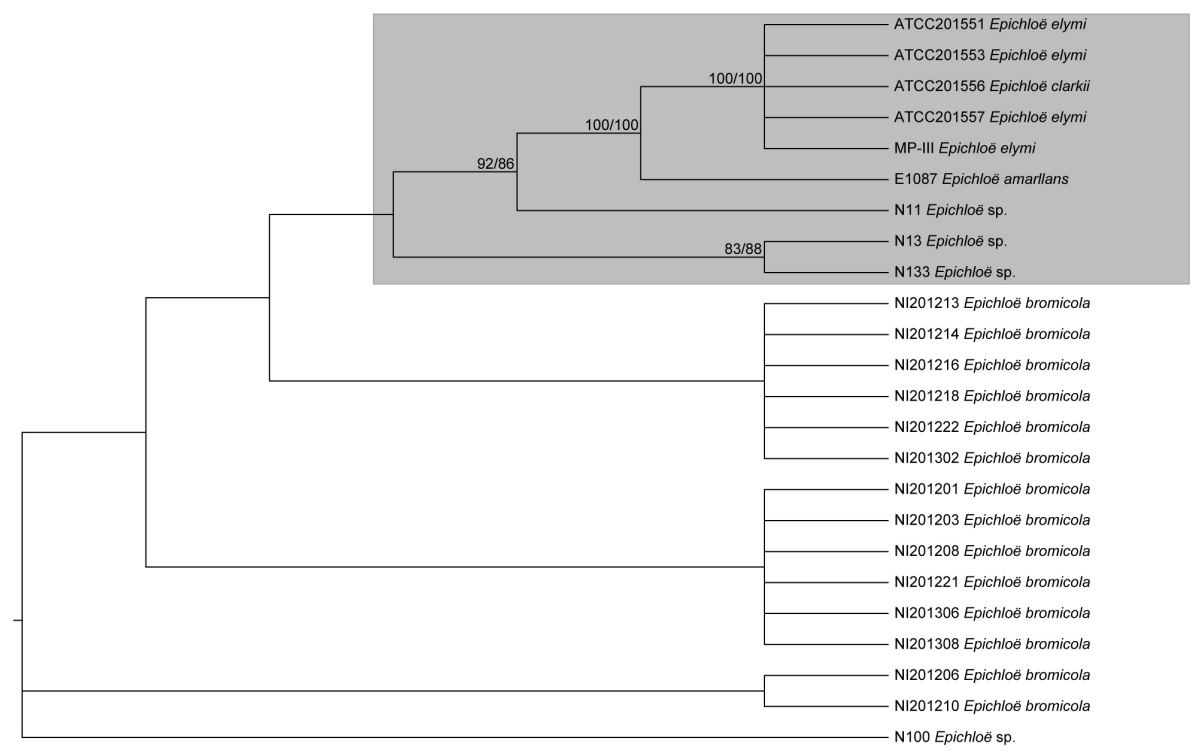

Figure 2. Maximum-parsimony tree of Epichloë endophytes based on tubB+tefA intron sequences. Bootstrap support values were calculated from 1000 replications. Branches with bootstrap values $>50 \%$ are shown. MP bootstrap values are listed first on each branch, followed by ML bootstrap values. The gray box contains a clade recovered in MP and ML analyses. 
Phylogenetic analyses of $\beta$-tubulin (tubB) and rDNA-ITS sequences (Moon et al., 2000) support the hypothesis that asexual Epichloë species evolved from sexually reproducing species that lost the sexual state. However, our results do not entirely support this sequence of events. Hordeum species are the potential diploid donors, and they may have formed earlier than Elymus species. Our results also suggest that isolates from Chinese Hordeum and North American Elymus species have the same origin, suggesting that sexual Epichloë endophytes from North American Elymus species are derived from asexual Epichloë endophytes from Chinese Hordeum species. Therefore, the most plausible explanation based on our analyses is that sexual Epichloë endophytes evolved from asexual Epichloë endophytes.

\section{Hypothesis of endophyte distribution based on host hybridization}

The artificial infection of endophyte-free plants (E-) with isolates from endophyteinfected plants $(E+)$ is used to improve biotic and abiotic resistance of plants (Leuchtmann and Clay, 1993). However, the success rate of this procedure is low. Based on our results, we hypothesize that endophytes may spread between grass hosts via plant hybridization. Our study provides preliminary evidence for this viewpoint in that the trees of grass hosts and endophytes suggested that sexual and asexual endophytes from North American and western Chinese Elymus species are most closely related to endophytes from Chinese Hordeum species. A previous study (Torabinejad and Mueller, 1993) found that the polyploid Elymus species in this study contain genomes of the St, $\mathrm{H}$ and $\mathrm{Y}$ types, potentially from Pseudoroegneria, Hordeum and an unknown species. Pseudoroegneria species do not contain endophytes (Card et al., 2014). The transmission of asexual Epichloë endophytes to the next generation generally occurs via seeds (White et al., 1993). Here, we hypothesize that Epichloë endophytes spread from Hordeum to Elymus via hybridization between Elymus and Hordeum species. We hope to find additional evidence for our hypothesis in a future study.

\section{Conflicts of interest}

The authors declare no conflict of interest.

\section{ACKNOWLEDGMENTS}

Research supported by grants from the National Basic Research Program of China (\#2014CB138702), the National Natural Science Foundation of China (\#31502001), and the Fundamental Research Funds for the Central Universities (Izujbky-2014-76, Izujbky-2014-81).

\section{REFERENCES}

Card SD, Faville MJ, Simpson WR, Johnson RD, et al. (2014). Mutualistic fungal endophytes in the Triticeae - survey and description. FEMS Microbiol. Ecol. 88: 94-106.

Clay K and Schardl CL (2002). Evolutionary origins and ecological consequences of endophyte symbiosis with grasses. Am. Nat. 160: S99-S127.

Dewey DR (1984). The genomic system of classification as a guide to intergeneric hybridization with the perennial Triticeae. In: Gene manipulation in plant improvement (Gustafson JP, ed.). Plenum Press, New York, 209-279.

Ekanayake PN, Hand ML, Spangenberg GC, Forster JW, et al. (2012). Genetic diversity and host specificity of fungal endophyte taxa in Fescue pasture grasses. Crop Sci. 52: 2243-2252. 
Gundel PE, Martínez-Ghersa MA, Omacini M, Cuyeu R, et al. (2012). Mutualism effectiveness and vertical transmission of symbiotic fungal endophytes in response to host genetic background. Evol. Appl. 5: 838-849.

Helfgott DM and Mason-Gamer RJ (2004). The evolution of North American Elymus (Triticeae, Poaceae) allotetraploids: evidence from phosphoenolpyruvate carboxylase gene sequences. Syst. Bot. 29: 850-861.

Jaaska V (1992). Isoenzyme variation in the grass genus Elymus (Poaceae). Hereditas 117: 11-22.

Jensen KB (1990). Cytology, fertility, and morphology of Elymuc Kengii (Keng) Tzvelev and E. grandiglumis (Keng) Á. Löve (Triticeae: Poaceae). Genome 33: 563-570.

Johnson LJ, de Bonth ACM, Briggs LR, Caradus JR, et al. (2013). The exploitation of epichloae endophytes for agricultural benefit. Fungal Divers. 60: 171-188.

Karimi S, Mirlohi A, Sabzalian MR, Sayed Tabatabaei BE, et al. (2012). Molecular evidence for Neotyphodium fungal endophyte variation and specificity with host grass species. Mycologia 104: 1281-1290.

Katoh K and Standley DM (2013). MAFFT multiple sequence alignment software version 7: improvements in performance and usability. Mol. Biol. Evol. 30: 772-780.

Leuchtmann A and Clay K (1993). Nonreciprocal compatibility between Epichloë and four host grasses. Mycologia 85: 157-163.

Linde-Laursen I, Seberg O and Salomon B (1994). Comparison of the Giemsa C-banded and N-banded karyotypes of two Elymus species, E. dentatus and E. glaucescens (Poaceae: Triticeae). Plant Syst. Evol. 192: 165-176.

Lu BR (1993). Genomic relationships within the Elymus parviglumis group (Triticeae: Poaceae). Plant Syst. Evol. 187: $191-211$.

Lu BR and Salömon B (1992). Differentiation of the SY genomes in Asiatic Elymus. Hereditas 116: 121-126.

Mason-Gamer RJ (2013). Phylogeny of a genomically diverse group of Elymus (Poaceae) allopolyploids reveals multiple levels of reticulation. PLoS One 8: e78449.

Mason-Gamer RJ and Orme NL (2002). Phylogenetic analysis of North American Elymus and the monogenomic Triticeae (Poaceae) using three choroplast DNA data sets. Genome 45: 991-1002.

Mason-Gamer RJ, Burns MM and Naum M (2010). Phylogenetic relationships and reticulation among Asian Elymus (Poaceae) allotetraploids: analyses of three nuclear gene trees. Mol. Phylogenet. Evol. 54: 10-22.

Moon CD, Scott B, Schardl CL and Christensen MJ (2000). The evolutionary origins of Epichloë endophytes from annual ryegrasses. Mycologia 92: 1103-1118.

Moon CD, Miles CO, Järlfors U and Schardl CL (2002). The evolutionary origins of three new Neotyphodium endophyte species from grasses indigenous to the Southern Hemisphere. Mycologia 94: 694-711.

Nicholas KB and Nicholas HB (1997). GeneDoc: a tool for editing and annotating multiple sequence alignments. Distributed by the author.

Nylander JAA (2004). MrModeltest v2. Program distributed by the author. Evolutionary Biology Centre, Uppsala University.

Schardl CL (2010). The Epichloae, symbionts of the grass subfamily Poöideae. Ann. Missouri Bot. Gard. 97: 646-665.

Schardl CL and Philips TD (1997). Protective grass endophytes. Where are they from and where are they going? Plant Dis. 81: $430-438$.

Schardl CL, Leuchtmann A, Chung KR, Penny D, et al. (1997). Coevolution by common descent of fungal symbionts (Epichloë spp.) and grass hosts. Mol. Biol. Evol. 14: 133-143.

Schardl CL, Craven KD, Speakman S, Stromberg A, et al. (2008). A novel test for host-symbiont codivergence indicates ancient origin of fungal endophytes in grasses. Syst. Bot. 57: 483-498.

Schardl CL, Young CA, Pan J, Florea S, et al. (2013). Currencies of mutualisms: sources of alkaloid genes in vertically transmitted epichloae. Toxins 5: 1064-1088.

Selosse MA and Schardl CL (2007). Fungal endophytes of grasses: hybrids rescued by vertical transmission? An evolutionary perspective. New Phytol. 173: 452-458.

Song $\mathrm{H}$ and Nan Z (2015). Origin, divergence, and phylogeny of asexual Epichloë endophyte in Elymus species from western China. PLoS One 10: e0127096.

Sun G (2002). Interspecific polymorphism at non-coding regions of chloroplast, mitochondrial DNA and rRNA IGS region in Elymus species. Hereditas 137: 119-124.

Sun G (2014). Molecular phylogeny revealed complex evolutionary process in Elymus species. J. Syst. Evol. 52: 706-711.

Swofford DL (2002). PAUP*: Phylogenetic analysis using parsimony ("and other methods). Version 4. Sinauer, Sunderland, Massachusetts, USA.

Taberlet P, Gielly L, Pautou G and Bouvet J (1991). Universal primers for amplification of three non-coding regions of chloroplast DNA. Plant Mol. Biol. 17: 1105-1109.

Torabinejad J and Mueller RJ (1993). Genome constitution of the Australian hexaploid grass Elymus scabrus (Poaceae: Triticeae). Genome 36: 147-151.

Vaidya G, Lohman DJ, Meier R (2011). SequenceMatrix: concatenation software for the fast assembly of multi-gene datasets with character set and codon information. Cladistics 27: 171-180. 
Wang RRC and Hsiao $\mathrm{C}$ (1986). Differentiation of $\mathrm{H}$ genomes of the genus Critesion: Evidence from synthetic hybrids involving Elymus and Critesion and one natural hybrid of C. violaceum and C. bogdanii. Can. J. Genet. Cytol. 28: 947-953.

White JF, Morgan-Jones G and Morrow AC (1993). Taxonomy, life cycle, reproduction and detection of Acremonium endophytes. Agr. Ecosyst. Environ. 44: 13-37.

Zhu MJ, Ren AZ, Wen W and Gao YB (2013). Diversity and taxonomy of endophytes from Leymus chinensis in the Inner Mongolia steppe of China. FEMS Microbiol. Lett. 340: 135-145. 